\title{
Road Infrastructure Heritage: From Scan to InfraBIM
}

\author{
VINCENZO BARRILE ${ }^{1 *}$, ERNESTO BERNARDO ${ }^{1}$, GABRIELE CANDELA ${ }^{1}$, GIULIANA \\ BILOTTA $^{2}$, ANTONINO MODAFFERI ${ }^{1}$, ANTONINO FOTIA ${ }^{1}$ \\ ${ }^{1}$ Geomatics Lab, Department of civil engineering, energy, environment and materials (DICEAM) \\ Mediterranea University of Reggio Calabria \\ Via Graziella Feo di Vito - 89124 Reggio Calabria \\ ITALY \\ 2 Dept. of Planning, \\ University IUAV of Venice, \\ Santa Croce 191, 30135, Venice
}

ITALY

\begin{abstract}
BIM is a methodology applied to the realization of design models applied to new buildings. To date, however, most of the building interventions as it happens in the field of cultural heritage are developed in the existing. For this reason, scan to BIM procedures are improved and improved every day to make the use of BIM easier. This document will describe the combination of different geomatics techniques used by the Geomatics Laboratory of the University of the Mediterranean in Reggio Calabria to create a Building Information Model of a highway viaduct (infraBIM). In particular, we paid more attention to the scan to BIM phase through the segmentation of the point cloud using machine learning techniques that allow to obtain the constitutive parametric elements of the 3D model. The model containing the geometric and physical data made available by the ANAS management body in order to use the potential of infraBIM. This methodology today is of particular importance for the control, monitoring, intervention, and maintenance of road infrastructures, optimizing the procedures existing up to now. The advantages would be even more evident considering that we are living in a particular historical moment, in which a large number of bridges and viaducts in our nation are subject to advanced forms of degradation.
\end{abstract}

Key-Words: - Road Infrastructure, BIM, InfraBIM, UAV, 3D, Parametric Model

Received: April 16, 2020. Revised: August 27, 2020. Re-revised: September 3, 2020.

Accepted: September 5, 2020. Published: September 9, 2020.

\section{Introduction}

\subsection{BIM and InfraBIM}

The organizations responsible for the maintenance of road structures (ANAS, Regions, Provinces, etc.) must face multiple and different problems depending on whether they are recent or dated structures. In the first case, executive projects and various information are available, so the aim of the surveys is to obtain a comparison with the project. In the second case, very often it is not possible to have design documents, so the important activity is also used to reconstruct the execution methods and break down the structural body into the elements that had been considered and sized in the design phase.

To date, monitoring of viaducts can take place through various SHM (Structural Health monitoring) solutions which follow two main monitoring logics:

1. Static monitoring system: vibrating wire strain gauges are used to monitor macro-sizes and infrastructure displacements of a few millimeters.

2. Dynamic monitoring system: accelerometers distributed on the deck and on the batteries are used to monitor the acceleration and the speed of vibration, in order to verify the ways in which the structure vibrates. This type of monitoring has the task of providing alerts in the event of exceeding preset thresholds, in order to avoid that the structure can overcome elastic non-return states.

Among the most used tools for the survey and modeling of these artifacts are UAVs and laser scanner technology, with integrated camera. The latter allows you to acquire large quantities of data, both geometric and photographic, in a short time. The choice of the laser scanner to perform a survey in a satisfactory manner, with adequate precision and completeness of representation, must take into account numerous considerations: precision, range, 
practical-operational characteristics, evaluation of the sockets necessary for complete visibility of all parts of the object to be surveyed, conditions in which one must operate to carry out the survey.

UAVs must take into account similar problems and although the photogrammetric survey is qualitatively lower than the survey with the laser scanner, the UAV allows to detect parts of the structure that would otherwise be inaccessible by the laser scanner [1 - 4].

To date, the use of terrestrial Mobile Mapping System [5;6], is widespread; it guarantees the possibility to acquired data for any threedimensional modeling and on it measurement systems and sensors are integrated; in this way it is possible to acquire simultaneously the quasicontinuous 3D position of the platform and georeferenced 2D / 3D metric data. For this purpose, from a technological point of view, an MMS is made up of integration of three main hardware components: optical sensors (laser scanner and / or digital cameras), navigation / positioning sensors (IMU / GNSS) and a control and synchronization unit.

A common element among all these applications is the fundamental importance of rigorous synchronization of the data flow through a high integration of the different measurement processes.

This generally allows to reduce the post-processing work and to provide real-time reliable estimates of the measurements made.

The precision and accuracy requirements for data acquired with MMS systems vary according to the application. However, both the parts relating to the acquisition and the parts relating to the management of MMS data by end users (public administrations, studies, superintendencies, etc.) are still too expensive.

In any case, regardless of the use of the systems adopted, the data and information acquired can be used for the construction of infrastructure models within the BIM (infraBIM).

BIM, Building Information Modeling is a very useful tool that has profoundly transformed the world of building and civil construction in all its different disciplines: architecture, engineering design, mechanical/hydraulic/electrical engineering, operation and maintenance, costs and safety, etc. [7 $-10]$.

Thanks to $\mathrm{BIM}$, to $2 \mathrm{D}$ and $3 \mathrm{D}$ assisted design, to parametric design and to the sharing and interoperability of the product model, we can conceive the work in all its phases, from concept to realization and consequently to the management of the work to be carried out or already made [11 - 13].
Although nowadays it is mainly used in the construction sector and MEP (mechanical, electrical and hydraulic engineering), it is still little used in the field of urban and infrastructure design (InfraBIM), where the processes and controls implemented by BIM can be managed effectively and at a higher level of efficiency. The term InfraBIM is used in reference to the adoption of the BIM methodology in the infrastructure sector. A sector that involves many aspects, from the analysis of the analyzed area to the relationships and interferences with other infrastructures. A multidisciplinary approach that consequently requires the correct implementation of the BIM methodology achieved by checking the consistency and quality of the information included in the models and which requires a strong disciplinary interconnection with the geographic information systems (GIS) [14 - 18]. This paper focuses on the BIM validation phase seen as a support for an adequate workflow: it not only allows to analyze the entire set of information associated with a parametric object, but also how the entire model meets the requirements of specific design. The BIM validation process in the design is not yet defined, but it is important to aim for a correct procedure in order to guarantee the quality of the design and the future management of the project.

Nowadays, developing BIM models means developing parametric 3D models with the aim of satisfying all design aspects at 360 degrees, and not just geometric ones. For this reason, the use of the BIM methodology requires further study on the new concept of "size" of the project. The 3D modeling with which it is possible to view the construction as a whole, and any object that constitutes it in a threedimensional way, allows to check for any interference present within the model (class detection) and to check that it complies with the project requests (code checking).

In 4D modeling, instead, the "time" variable is added to the previous modeling level, in order to plan time management, relative to the entire life cycle of the project. We can interpret it as a sort of project management: time and planning information are connected to each object of the model, in order to reduce the interference deriving from the various activities that occur during the life of the work.

Therefore, a BIM model can be associated with various information that also determines the performance level of the work itself, allowing to manage and maintain the building object and all its components during the life cycle.

The process of creating digital models for infrastructural works involves a series of 
multidisciplinary activities, which require the use of different formats for data processing. Creating a complete 3D digital model of the work means clearly and neatly including the aspects relating to costs, times and finally management of maintenance.

The Geomatics techniques of data acquisition for the reconstruction of point clouds are well suited for the implementation and realization of purely geometric models.

\section{Territorial frameworks, survey by UAV and 3D}

We applied the described methodology to a viaduct located on A2 highway. Annunziata viaduct is on "Autostrada del Mediterraneo" in the city of Reggio Calabria, Italy (Fig.1). The viaduct, built on 1970 upon the "Annunziata" river, is in a high seismic risk zone (in this regard, a monitoring network has been set up in that area that can continuously acquire displacement data, given the importance of the infrastructure).

This viaduct is located near the city center and therefore has a high concentration of vehicular traffic especially at peak times.

Given its strategic importance within the urban fabric, the same has been the subject of multiple studies and analyzes, and interventions given the strong deterioration at the spans.

The Annunziata viaduct is made of pre-stressed reinforced concrete with 9 short-spans of $27 \mathrm{~m}$ (the two bridges one for each direction), and a total length of $250 \mathrm{~m}$ (when cornering). The radius of curvature is $352 \mathrm{~m}$ for a height of $25 \mathrm{~m}$. The two bridges are supported by a couple of piers with a common base. The viaduct is located in an area at high risk of undermining the foot of the pillars given the irregular flow of the stream flow itself. The spans of the viaduct arise on soft rocky terrain.

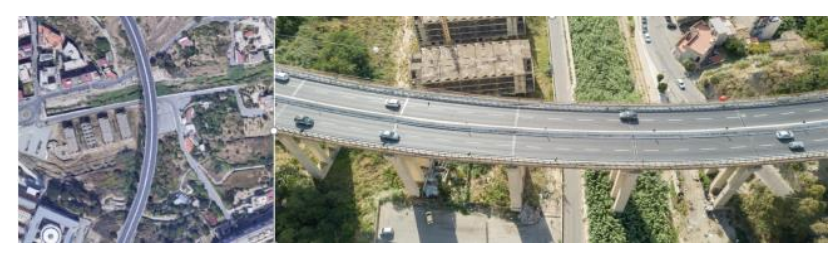

Fig. 1 Territorial framework

The documentation relating the viaduct was provided by ANAS S.p.A.. The history of the viaduct was reconstructed on the basis of archival research and information collected from the Reggio Calabria compartment.
Thanks to the photogrammetric principles with a low-cost UAV, No.1039 photos were taken from different perspectives, acquiring a data set that allowed analyzing the structure. Agisoft Metashape allowed processing these photos according to the principles described above and obtaining a $3 \mathrm{D}$ point cloud, thanks to SfM and MVS, a scattered point cloud of approximately 70,000 points and a dense cloud of approximately 4,000,000 are created (Fig.2).
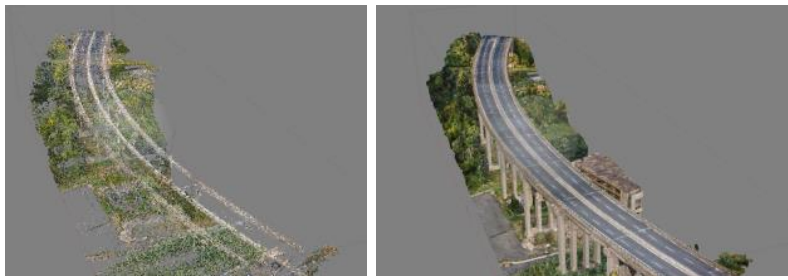

Fig. 2 Scattered cloud and 3D model

\section{From scan to infraBIM}

In order to extract relevant information from the point cloud, it is necessary to segment and classify the object concerned within the appropriate scene.

For bridge and viaduct, omitting the 3D segmentation made directly from the cloud points, automatic learning based on the analysis of 2D images can provide good results.

To date there are many techniques and algorithms to treat data based on:

- segmentation: a process that groups together several homogeneous point clouds with similar properties (geometric, radiometric etc.), [19].

- classification: which defines and assigns points in specific classes called "labels" according to different judgments.

For example, for the segmentation phase we remember, Edge-based segmentation [20] relies on algorithms to identify edges and contours of different regions and to group points within borders to provide final segments. Segmentation by model adaptation is based on the observation of manmade objects that can be broken down into geometric primitives such as planes, spheres and cylinders. The primitive forms are then inserted in the cloud of points which conform to the mathematical representation are labeled as a segment. In the hybrid method, multiple combined methods are used to harness the strength of one method and avoid the weakness of another. In particular, the range image of $3 \mathrm{D}$ objects is divided into surface primitives which are homogeneous in their intrinsic differential geometric properties and do not contain discontinuities in either depth of 
surface orientation. The method is based on the computation of partial derivatives, obtained by a selective local biquadratic surface fit. Then, by computing the Gaussian and mean curvatures, an initial region-based segmentation is obtained in the form of a curvature sign map. Two additional initial edge-based segmentations are also computed from the partial derivatives and depth values, namely, jump and roof-edge maps. The three image maps are then combined to produce the final segmentation [21].

Accuracy depends on the methods used and the scene where the objects are labeled.

Finally, machine learning methods are based on machine learning of algorithms (including deep learning neural network) [22]. Machine learning is an artificial discipline that uses artificial intelligence algorithms and allows the computer to make decisions based on empirical models and saved data. The learning that allows the computer to make a decision can be supervised (reinforcement learning) or without supervision. The difference lies in the fact that where there is no supervision, we try to determine how the data are, while in supervised learning the data is labeled so that the machine performs a task correctly. Machine Learning methods use a weakly - and semi - supervised learning of a Deep Convolutional Neural Network (DCNN) for semantic image segmentation. It use bounding box and image-level labels as markup training data on the basis of DeepLab and used the Expected Maximization (EM) algorithm to estimate unmarked Pixel class and Convolutional Neural Networks (CNN) parameters. DeepLab method is divided into two steps, the first step is still using the Fully Convolutional Neural Network (FCN) to get the coarse score map and interpolate to the original image size, and then the second step began to borrow the fully connected Conditional Random Field (CRF) from the FCN to get the details of the segmentation refinement.

The revision of machine learning techniques applied to segmentation of images and 3D data is presented in [23]. The dataset for 3D segmentation via Neural Network developed by Stanford University is more precise and broader.

The classification of each segmented point can be obtained using three different approaches:

- supervised approach, where semantic categories are learned from an annotated dataset, and the trained model is used to provide a classification of the whole dataset
- unsupervised approach, in which the data are automatically partitioned into segments based on user-supplied parameters

- interactive approach, in which the user is actively involved in the segmentation/classification cycle by guiding the extraction of segments through feedback.

In the specific case, we used a elaborate procedure based on the segmentation of individual images and on the transfer of 2D segmentation on the 3D point cloud through the masks.

Segmentation was performed using Mask-RCNN (convolutional neural network). This network can be used to automatically segment and build pixel masks for each object in an image: in an image the model is trained to perform the structure, recognize and divide the different structural parts. Mask R-CNN (Mask Region-based Convolutional Neural Networks) is an extension of the Faster RCNN model for object detection, localization and instance segmentation in natural images. The principal components of Mask R-CNN are: Pyramid CNN, Region Proposal Network, RoIAlign Network Head.

1) Backbone Network a Pyramid $\mathrm{CNN}$ used to extract high level visual features from the entire image.

2) Region Proposal Network, that is a fully convolutional network that takes in image features from the backbone network and proposes candidate object bounding boxes with their objectness score.

3) RoIAlign: In order to predict pixel masks accurately Mask R-CNN requires the RoI features (which are small feature maps) to be well aligned to accurately preserve the per-pixel spatial correspondence. For this purpose Mask $\mathrm{R}-\mathrm{CNN}$ replaced the RoIPool layer in Faster RCNN with an RoIAlign layer. The RoIAlign layer uses bi-linear interpolation to compute the exact values of the input features at four regularly sampled locations in each RoI bin, and then performs max or average pooling on the features.

4) Network Head (Bounding Box Regression, Classification and Mask Prediction): Finally, the RoIAligned features are passed to the network head which performs three parallel tasks of bounding box regression, classification and mask prediction. The classifier and regressor output the class labels and bounding box offsets collapsing the RoIAligned features into short output vectors by fully-connected (fc) layers. 
The mask branch predicts an $\mathrm{m} \times \mathrm{m}$ mask from each RoI using a Fully Convolutional Network $(\mathrm{FCN})$. This allows each layer in the mask branch to maintain the explicit $\mathrm{m} \times \mathrm{m}$ object spatial layout without collapsing it into a vector representation that lacks spatial dimensions.

It is good to remember data sets and features play an important role in the use of machine learning because these methods are based on the quantity and quality of the data input.

In the specific case, the training of the network took place by uploading the set of images on compilers that exploit the methodology used (Mask - RCNN) by implementing the dataset manually (attempts will be made to automate the process). This possibility allows us to independently decide whether an image should be accepted or not, (therefore the choice of images remains at the user's discretion).

The neural network is so applied to the complete data set to obtain masks for each selected element, such as stacks and decks, as shown in the Fig. 3 - 4.
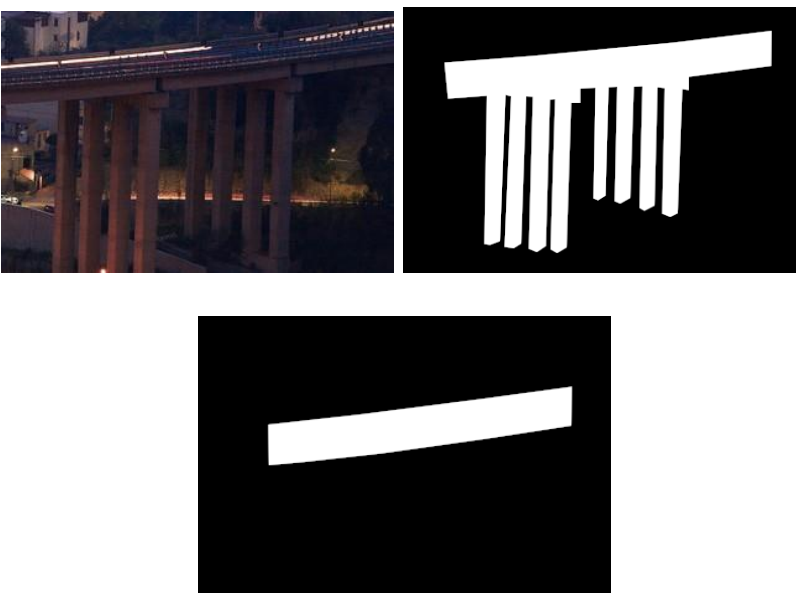

Fig. 3 Viaduct and segmentations

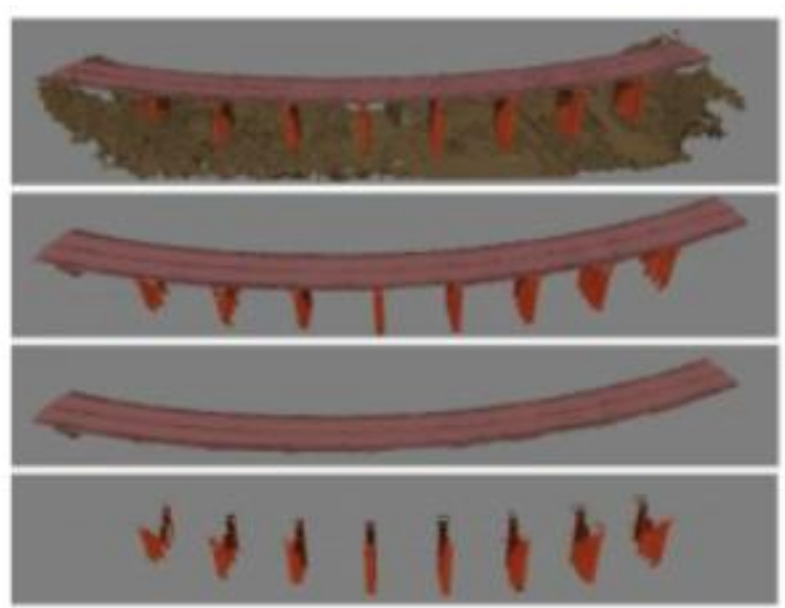

Fig. 4 Segmentations and classification of cloud elements

Once the previous phases were completed, it was possible to start modeling the parametric families of the elements constituting the viaduct.

After completing the segmentation phases of the objects, it is possible to proceed with their modeling. This type of processing must be carried out manually portion by portion, and therefore allows to create surfaces. Clearly, modeling is done by processing each portion of the point cloud from time to time.

Consistent with its methodological logic, Building Information Modeling allows to characterize all the elements of the infrastructure model by defining classes of objects.

Given the heterogeneity of the data that distinguishes the digital modeling of an infrastructure in the BIM environment, the issue of interoperability - and in particular of a complete and integral data retention - is particularly important in this area.

A structure of this type naturally presupposes the subdivision of the infrastructure into a certain number of components and their association with the respective information content. From the BIM model it is of course possible all the necessary project documents, plans, elevations and sections, which as is proper to the BIM environment are dynamically updated according to the changes made to the model.

The structure of the viaduct consists of several main elements, each of which is modeled separately as a "family" and all is then assembled in order to be able to appreciate the work in its entirety.

In particular, part of the process was the creation of the different families that make up the Revit model. Families are both 2D and 3D elements united by parametric properties that influence their graphic representation. In fact, in addition to the definition of the different materials, the possibility of making modifications and that these are applied automatically to the global model is of particular importance. In Revit there are several basic types of creation, they have chosen:

- Mass families: this type of family was useful for creating the profiles of the mixed steel-concrete superstructure. In these models, sections can be created that adapt according to the change of parameters.

- Adaptive families: In this case these families have been used for the correct positioning in the global model, parameters can be inserted that 
allow the correct position of the works with respect to other elements making up the model. This is a fundamental aspect because any changes applied to individual objects must be implemented by the global model without causing errors or conflicts between the elements.

Another important function that Revit offers is the possibility of using nested families, i.e. families internal to other families. In this way, for example, the $2 \mathrm{D}$ sections were created as a mass family and then loaded into an adaptive family model so as to control the position in relation to other elements.

These elements were imported as individual elements in the BIM software (Autodesk Revit) as IFC file, using the entire cloud as a guideline for the placement of the various objects (Fig. 5). This process guarantees to obtain the appearance geometric and material closer to the real conformation of each component (ensuring also an information management).
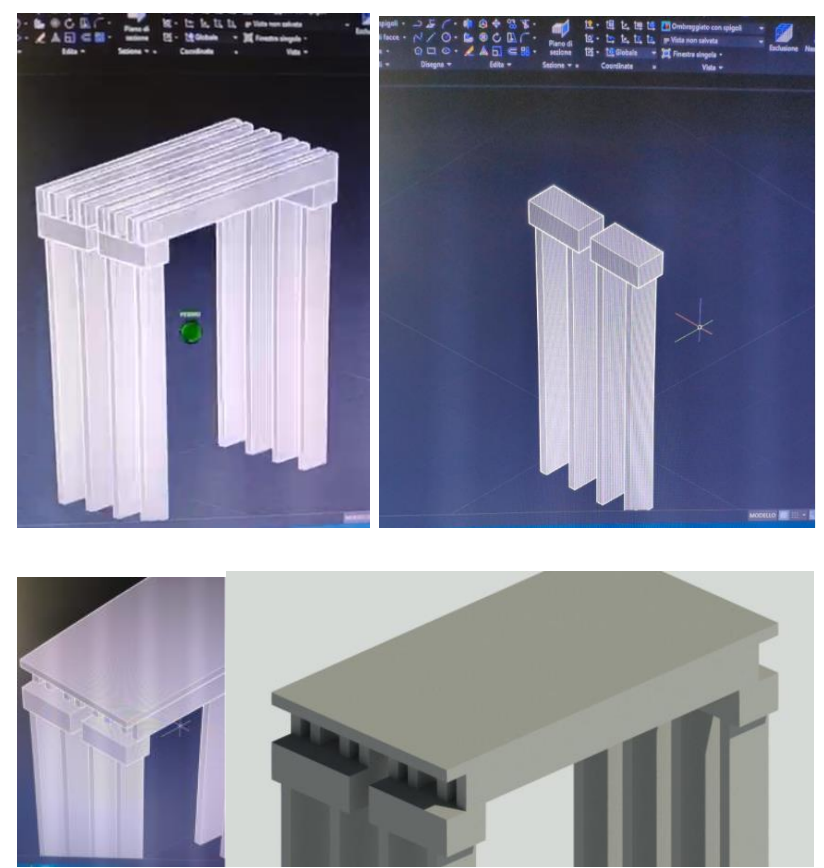

Fig. 5 Elements in BIM

\section{Database implementation}

Once the parametric elements have been created, the implementation of infrastructure database is achieved by attributing the acquired data.

Applying the BIM methodology to an infrastructure means creating digital models complete with all the information available and that can be regularly updated, in order to allow the actors involved in the construction process to always have a true picture of the situation available.
The created 3D parametric model so far allows an excellent overall view of the construction elements of the Annunziata viaduct and of framing the territorial context in which it is located, but the parameters created relate only to the geometric data. For this reason, we decided to fully exploit the potential of infraBIM, going to insert all the data and parameters obtained from the various execution and maintenance projects of the viaduct [24]. So, we can guarantee perfect interoperability between technicians. In this case, the database was completed by entering all the information available and necessary for the maintenance of the work. In particular, the geometric dimensions of the structure and the roadway were indicated, the periods of maintenance interventions were included as well as the type of intervention. Accidents were recorded, and the consequent structural tests (if carried out) [25-29]; the level of efficiency and the variation in average daily traffic over time, the presence of horizontal and vertical signs and the dates of application were indicated, a particular system was also implemented which, according to the type of material used for each element, of the degree of exposure of the work and from the level of salinity present, it is able to return the level of degradation (as shown in Fig. 6). 

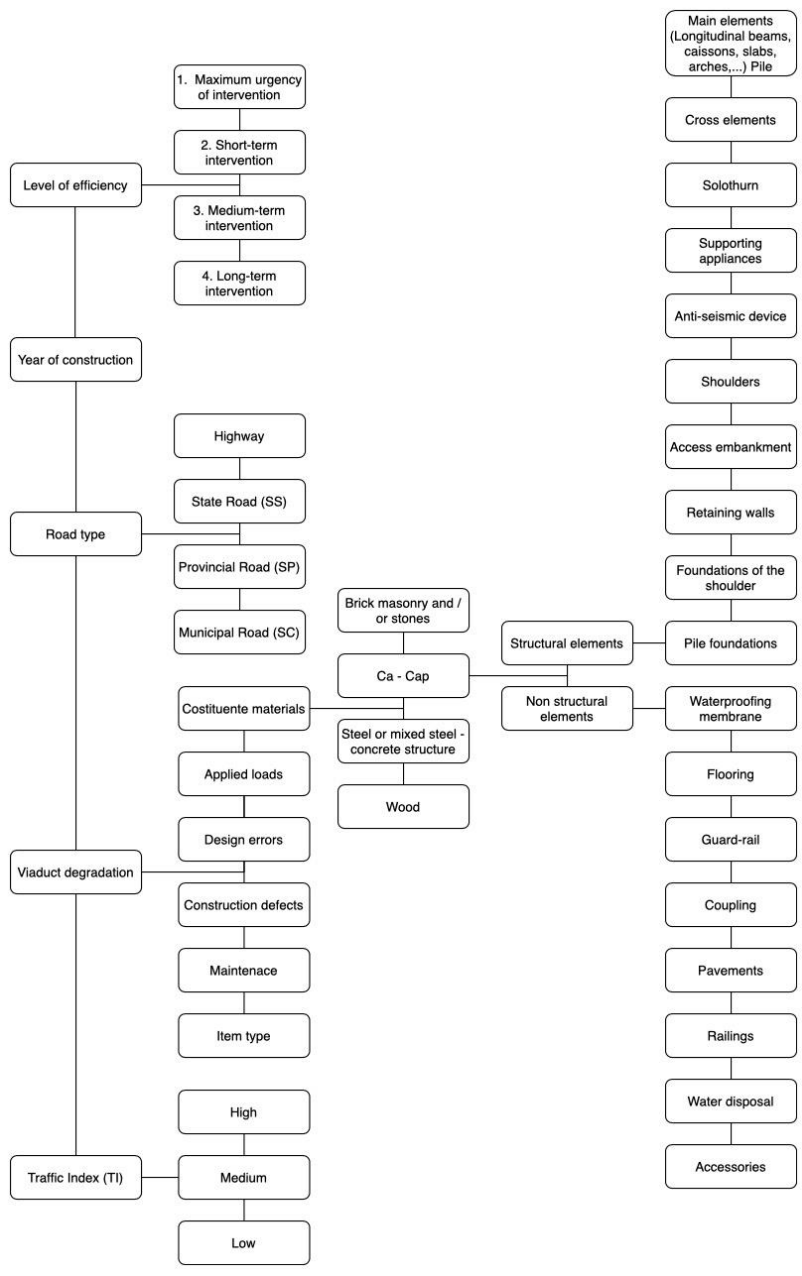

Fig. 6 database implementation

We can obtain a comparison with the project, obtain the structural behavior under loads (static, mobile) due to ever larger and heavier vehicles, seismic actions, maintenance plans in order to identify the critical parts of the structure on which to intervene; for the identification of degraded areas or areas with detached coverings, on which it is necessary to intervene with recovery and restoration actions; for the identification of the vibrating modes of the structure independent of external stresses, necessary to predict its behavior under dynamic loads.

In this way, infraBIM is used as a digitization tool to support information sharing and management. In this regard, it can be observed that in literature the BIM can be understood as an "information container" in which to insert graphic data (such as drawings) and specific technical attributes (such as technical data sheets and characteristics) also relating to the expected life cycle, which helps manage the projects of each discipline in a single data sharing environment ensuring the traceability of all the activities carried out. In fact, BIM guarantees collaboration between designers and integration between processes. BIM is therefore a collaborative design method as it allows you to integrate useful information at each stage of the design into a single model: architectural, structural, plant, energy and management. For this reason, it can be used by installers, structural engineers, architects, builders, fitters, testers etc. The threedimensional model therefore contains information regarding volume and size, material, appearance, technical characteristics that are not lost in communication to other studies and other IT platforms. In addition, a BIM project gives the client the opportunity to have a virtual elaboration of the life cycle of the building, even after the design phase; in this way it is easier to monitor the age of the materials and better plan the maintenance.

\section{Discussion}

The creation of BIM models from geomatic techniques therefore allows to shift the attention from the buildable to the built. Just as in the cultural heritage field, it is also much easier in the infrastructure sector to face maintenance and conservation rather than new buildings. Then it becomes crucial at the time of the IOT to carry out interventions aimed at creating a "virtual library of the work" where the various interventions that followed one another, the different materials used are noted, so as to be able to intelligently program the interventions of maintenance required.

To date, segmentation processes can be developed directly on images (2D segmentation) or directly on three-dimensional models (3D segmentation). Among the various segmentation processes, the semantic segmentation of point clouds is among the most known problems.

In fact, the last few years have seen the development of algorithms and methodologies in order to reduce human intervention for two of the most common point cloud processing tasks: first, reconstruction and abstraction of the surface, and second, recognition of objects and semantic understanding of the scene. However, these activities are still a pending research topic and, in the fields applied, the processing of the point cloud remains at least partly manual. The final objective therefore remains to identify the belonging to the class of each 3D point. This problem is partially related to $2 \mathrm{D}$ semantic segmentation, where the goal is to label each pixel of the image.

Through the use of a deep learning framework, it is possible to learn not only the classifier, but also the representation of the characteristics. 
In the specific case, we wanted to use systems based on deep neural networks in image processing for classification or semantic segmentation based directly on the images that will subsequently make up the 3D model (thus not limiting the problem of operator intervention in the phase learning of the neural network and therefore increasing the processing time).

To date there are only a few proposals on these 3D tasks, since those voxel-based approaches besides requiring a lot of memory are methods that work directly on sets of unordered points, using architectures with completely connected levels and consequently it would be necessary to process more models 3D, clearly generating classes for the entire 3D shape.

Comparing the different methods of 2D segmentation, the use of Mask-RCNN has been shown to have higher convergence speeds than other applied solutions. This is because the functions related to the identification of a basic element can be learned in the first layers of the network. By using additional layers, it does not improve detection accuracy, but rather slows down convergence. Clearly, with other systems, by increasing the layers, on the one hand a more performing response is obtained, but on the other, computational times are increased.

\section{Conclusions}

We are going through a historical period in which the age and the state of affairs of Italy's infrastructural heritage require the definition of scheduled maintenance actions in order to preserve its value and ensure its safety conditions. The BIM (Building Information Modeling) methodology is seen as a potential tool to overcome productivity problems in the construction sector. The BIM methodology applied to Infrastructure design can represent an improvement in the workflow, the project and the final product. In the world of infrastructures there is a need to make different specialist disciplines dialogue, for example the discipline of road design with the structural one.

Furthermore, infrastructure projects are often divided between different design groups and the optimization of communication between groups is a fundamental aspect for the overall improvement of the project itself. BIM therefore aims to provide effective means of adequately integrating the multidisciplinary information required for the various project phases: planning, design, construction and maintenance. However, to date infraBIM is used only for new designs, yet maintenance plans are continuously necessary at the present day. For this reason, as it also happens for HBIM, the note proposed an alternative methodology that sees the combination of multiple techniques for the parameterization of the infrastructure. In particular, we move from the reaction of a cloud of points to its segmentation and finally to the BIM modeling, all accompanied by the insertion of the acquired and digitized information.

\section{References:}

[1] Artese, S., Miceli, A., Talarico, P., Venneri, A., Zagari, G., Zinno, R., Ponti antichi e moderni: utilizzo di tecniche geomatiche per il rilievo, la rappresentazione e la modellazione strutturale, Asita, 2015, pp.797-804.

[2] Barrile, V., Candela, G., Fotia, A., Bernardo, E., UAV Survey of Bridges and Viaduct: Workflow and Application, In Lecture Notes in Computer Science (Including Subseries Lecture Notes in Artificial Intelligence and Lecture Notes in Bioinformatics), Springer: Cham, Switzerland, 2019, pp. 195-199. https://doi.org/10.1007/978-3-030-24305-0_21

[3] Piras, M., Di Pietra, V., Visintini, D., 3D modeling of industrial heritage building using COTs system: test, limits and performances, International Archives of the Photogrammetry, Remote Sensing and Spatial Information Sciences, Vol.XLII-2/W6, 2017, pp. 281-288. https://doi.org/10.5194/isprs-archives-XLII-2W6-281-2017

[4] Barrile, V., Candela, G., Fotia, A., Bernardo, E., UAV Survey of Bridges and Viaduct: Workflow and Application, Misra S. et al. (eds) Computational Science and Its Applications ICCSA, 2019, pp.269-284. Lecture Notes in Computer Science, Springer, Cham, Vol.11622. https://doi.org/10.1007/978-3-030-24305-0 21

[5] Mancini, A., Malinverni, E. S., Frontoni, E., Zingaretti, P., Road pavement crack automatic detection by MMS images, 21st Mediterranean Conference on Control and Automation, IEEE, Chania, 2013, pp. 1589-1596. doi: 10.1109/MED.2013.6608934

[6] Florkova, Z., Duris, L., Veselovsky, M., Sedivý S., and Kovalova, D., Three-dimensional mobile mapping system and its use in road engineering, Matec Web Conf., Vol.196, No. 04082, 2018.

https://doi.org/10.1051/matecconf/2018196040 $\underline{82}$

[7] McGraw-Hill Construction, Bernstein, H. M., Jones, S. A., \& Gudgel, J. E., The Business 
Value of BIM in Europe: Getting Building Information Modeling to the Bottom Line in the United Kingdom, France and Germany, Design and Construction Intelligence SmartMarket Report, McGraw-Hill Construction, 2010.

[8] Jones, S. A., \& Bernstein, H. M., The Business Value of BIM for Infrastructure Addressing America's Infrastructure Challenges with Collaboration and Technology, Design and Construction Intelligence SmartMarket Report, McGraw-Hill Construction, 2012.

[9] Azhar, S., Building Information Modeling (BIM): Trends, Benefits, Risks, and Challenges for the AEC Industry, Leadership and Management in Engineering, Vol.11, Issue 3, 2011, pp. 241-252.

https://doi.org/10.1061/(ASCE)LM.19435630.0000127

[10] Morin, G.; Hassall, S.; \& Chandler, R., Case study - The real life benefits of Geotechnical Building Information Modelling, Information Technology in Geo-Engineering, D.G. Toll et al., IOS Press, 2014, pp. 95-102.

doi: $\underline{10.3233 / 978-1-61499-417-6-95}$

[11] Bae, H., Golparvar-Fard, M., White, J, Highprecision vision-based mobile augmented reality system for contextaware architectural, engineering, construction and facility management (AEC/FM) applications. Visual. Eng., Vol.1, No. 3, 2013. https://doi.org/10.1186/2213-7459-1-3

[12] Barrile, V., Fotia A., Bilotta G., Geomatics and augmented reality experiments for the cultural heritage, Applied Geomatics, Vol.10, Issue 4, 2018, pp. 569-578.

https://doi.org/10.1007/s12518-018-0231-5

[13] Barrile, V., Fotia, A., Bilotta, G., De Carlo, D., Integration of geomatics methodologies and creation of a cultural heritage app using augmented reality. Virtual Archaeology Review, Vol.10, No.20, 2019.

https://doi.org/10.4995/var.2019.10361

[14] Bradley, A., Li, H., Lark, R., Dunn, S., BIM for infrastructure: An overall review and constructor perspective, Automation in Construction, Vol. 71, Part. 2, 2016, pp. 139152.

https://doi.org/10.1016/j.autcon.2016.08.019

[15] Biancardo, S. A., Zhang, W., Coraggio, G., BIM Reverse Engineering: Digital Transformation of Existing Roads, ICTTE 2018 - The International Conference on Traffic and
Transport Engineering, Belgrade, Serbia, pp. 584-591, Isbn 978-86-916153-4-5.

[16] Lee, N., Salama, T., Wang, G., Building Information Modeling for Quality Management in Infrastructure Construction Projects. Computing in Civil and Building Engineering: Proceedings of the 2014 International Conference on Computing in Civil and Building Engineering, 2014, pp. 65-72. https://doi.org/10.1061/9780784413616.009

[17] Leone, M., D'Andrea, A., Loprencipe, G., Malavasi, G., Bernardini, L., Building Information Modeling (BIM): Prospects for the development of railway infrastructure industry, Transport Infrastructure and Systems: Proceedings of the AIIT International Congress on Transport Infrastructure and Systems, Tis 2017, pp. 547-553.

[18] Marzouk, M., Hisham, M., Ismail, S., Youssef, M., Seif, O., On the use of Building Information Modeling in infrastructure bridges. Proceedings of the 27th International Conference on Applications of IT in the AEC Industry, Cairo, Egypt, 2010, pp. 1-10.

[19] Barrile, V., Candela, G., Fotia, A., Point cloud segmentation using image processing techniques for structural analysis. ISPRS Ann. Photogramm. Remote Sens. Spat. Inf. Sci. 2019, pp. 187-193.

https://doi.org/10.5194/isprs-archives-XLII-2W11-187-2019

[20] Rabbani, T., van den Heuvel, F. A., \& Vosselman, G., Segmentation of point clouds using smoothness constraints, Isprs: Proceedings of the ISPRS commission $V$ symposium: image engineering and vision metrology, Dresden, Germany, Vol.35, Part 6, 2006, pp. 248-253.

[21] Yokoya, N., and Levine, M. D., Range image segmentation based on differential geometry: a hybrid approach, in IEEE Transactions on Pattern Analysis and Machine Intelligence, Vol.11, No.6, 1989, pp. 643-649. doi: $10.1109 / 34.24798$

[22] Zeiler, M. D., Fergus, R., Visualizing and Understanding Convolutional Networks. In: Fleet D., Pajdla T., Schiele B., Tuytelaars T. (eds) Computer Vision - ECCV 2014. Lecture Notes in Computer Science, Springer, Cham, Vol. 8689, 2014.

https://doi.org/10.1007/978-3-319-10590-1_53

[23] Garcia - Garcia, A., Orts-Escolano S., Oprea S.; Villena-Martinez, V., Garcia-Rodriguez J., A Review on Deep Learning Techniques 
Applied to Semantic Segmentation, arXiv:1704.06857 [cs.CV], 2017.

[24] Bernardello R. A., Il Building Information Modeling per la modellizzazione di ponti e viadotti, ICEA, 2019.

[25] Fukuda, Y., Feng, M.Q., Narita, Y., Kaneko, S., Tanaka, T., Vision-based displacement sensor for monitoring dynamic response using robust object search algorithm, IEEE Sens. J., Vol.13, No. 12, 2013, pp. 4725-4732. doi: 10.1109/JSEN.2013.2273309

[26] Pucinotti, R., Fiordaliso, G., Multi-Span SteelConcrete Bridges with Anti-Seismic Devices: A Case Study, Front. Built Environ, 72, Vol.5, 2019, pp. 1-15.

https://doi.org/10.3389/fbuil.2019.00072

[27] Cristofaro, M. T., Pucinotti, R., Tanganelli, M., De Stefano, M., The dispersion of concrete compressive strength of existing buildings, Geotech. Geol. Earthq. Eng, Vol. 33, 2014, pp. 275-285.

https://doi.org/10.1007/978-3-319-06394-2 16

[28] Pucinotti, R., Reinforced concrete structure: Non destructive in situ strength assessment of concrete. Constr. Build. Mater, Vol. 75, 2015, pp. 331-341.

https://doi.org/10.1016/j.conbuildmat.2014.11. $\underline{023}$

[29] Pucinotti, R., Assessment of in situ characteristic concrete strength, Constr. Build. Mater, Vol. 44, 2013, pp. 63-73.

https://doi.org/10.1016/j.conbuildmat.2013.02. $\underline{041}$

\section{Creative Commons Attribution License 4.0 (Attribution 4.0 International, CC BY 4.0)}

This article is published under the terms of the Creative Commons Attribution License 4.0

https://creativecommons.org/licenses/by/4.0/deed.en_US 